\title{
WATER FORMATION IN THE UPPER ATMOSPHERE OF THE EARLY EARTH
}

\author{
Benjamin Fleury ${ }^{1}$, Nathalie Carrasco ${ }^{1,2}$, Emmanuel MarcQ ${ }^{1}$, Ludovic Vettier ${ }^{1}$, and Anni Mä̈̈ttänen ${ }^{1}$ \\ ${ }^{1}$ Université Versailles St-Quentin; Sorbonne Universités, UPMC Univ. Paris 06; CNRS/INSU, LATMOS-IPSL, \\ 11 Boulevard d'Alembert, F-78280 Guyancourt, France; benjamin.fleury@latmos.ipsl.fr \\ ${ }^{2}$ Institut Universitaire de France, 103 Bd St-Michel, F-75005 Paris, France \\ Received 2014 December 30; accepted 2015 June 17; published 2015 July 10
}

\begin{abstract}
The water concentration and distribution in the early Earth's atmosphere are important parameters that contribute to the chemistry and the radiative budget of the atmosphere. If the atmosphere above the troposphere is generally considered as dry, photochemistry is known to be responsible for the production of numerous minor species. Here we used an experimental setup to study the production of water in conditions simulating the chemistry above the troposphere of the early Earth with an atmospheric composition based on three major molecules: $\mathrm{N}_{2}, \mathrm{CO}_{2}$, and $\mathrm{H}_{2}$. The formation of gaseous products was monitored using infrared spectroscopy. Water was found as the major product, with approximately $10 \%$ of the gas products detected. This important water formation is discussed in the context of the early Earth.
\end{abstract}

Key words: astrochemistry - Earth - planets and satellites: atmospheres

\section{INTRODUCTION}

Determining the environment of the Earth before the apparition of the life is of prime interest to determine criteria of habitability. The atmospheric water content is one of the elements that determines the environment of a planetary body. As explained in Wordsworth \& Pierrehumbert (2013), vertical water distribution in terrestrial planet atmospheres is usually considered as dominated by transport phenomena from the surface up to the atmosphere where water can be photolyzed by solar UV. This transport involves a hydrogen escape and oxidation of the crust. It is, however, supposed to be limited by a cold trapping of water in the troposphere. Indeed, if the conditions of pressure and temperature are compatible, the colder upper region of the troposphere involves a condensation of water. This process traps water in the troposphere, and the atmospheric layers above are considered as dry.

The aim of this study is to experimentally investigate whether atmospheric photochemistry at high altitudes could be a source of water in layers where $\mathrm{CO}_{2}$ is photodissociated (stratosphere/mesosphere) and ionized (thermosphere). We consider an early atmosphere dominated by $\mathrm{N}_{2}$ and $\mathrm{CO}_{2}$ (Marty et al. 2013), with $\mathrm{H}_{2}$ as a compliment according to Kasting (1993) and Kuramoto et al. (2013). To address this question, we use an experimental setup called PAMPRE to study and quantify the production of water in conditions simulating the photoactive atmospheric layers of the early Earth and discuss the impact on the primitive Earth.

\section{EXPERIMENTAL SETUP AND ANALYTICAL PROTOCOLS}

\subsection{PAMPRE Experiment}

The PAMPRE experimental setup has been described in detail previously (Szopa et al. 2006; Alcouffe et al. 2010). It is a radio-frequency capacitively coupled plasma (RF CCP) at a low pressure. In this experiment, a discharge is generated between a polarized electrode and a cylindrical grid grounded electrode confining the plasma. Before each experiment, the reactor is heated and pumped down to $2 \cdot 10^{-6}$ mbar. A gaseous flow is injected continuously and pumped through a rotary vane vacuum pump. Three gas bottles are used to generate the reactive mixture: one with a high-purity of $\mathrm{N}_{2}(99,999 \%)$, one containing a $\mathrm{N}_{2}-\mathrm{H}_{2}$ mixture with $5 \%$ of $\mathrm{H}_{2}$, and one with $\mathrm{CO}_{2}$ $(99,995 \%)$. The generator RF power is set to $30 \mathrm{~W}$ and the total gas flow rate to $55 \mathrm{sccm}$ resulting in a $0.9 \mathrm{mbar}$ pressure in the reactor. The initial hydrogen mixing ratio is kept constant at $4 \%$ for all experiments. This concentration is chosen in agreement with recent modeling of $\mathrm{H}_{2}$ mixing ratio giving an upper limit about $1 \%$ for the Archean atmosphere (Kuramoto et al. 2013). The gas flow is adjusted from an experiment to another to introduce carbon dioxide at various mixing ratios ranging from $1 \%$ to $10 \%$. Compositions of the different gaseous mixtures studied here are given in Table 1.

First, a series of experiments is done at room temperature to monitor the $\mathrm{CO}_{2}$ consumption in the plasma by in situ mass spectrometry. Second, in order to detect and identify gaseous species produced in low quantity, a new series of experiments is realized using cold trapping of these species on the plasma box. We used a continuous injection of liquid nitrogen $\left(\mathrm{L}_{\mathrm{N}_{2}}\right)$ inside the stainless-steel block supporting the grounded electrode. The plasma box is cooled by thermal conduction. The temperature is fixed at $173 \mathrm{~K}$ to prevent $\mathrm{N}_{2}, \mathrm{H}_{2}$ and $\mathrm{CO}_{2}$ condensation. Products are accumulated during four hours of plasma duration. After plasma is turned off, the reactor is pumped to eliminate reactive species. The reactor is isolated and the plasma box is warmed up to room temperature to release the trapped gases. Then the gaseous phase is analyzed in situ by infrared spectroscopy.

\subsection{Direct In Situ Mass Spectrometry on the Gaseous Phase}

In situ measurements of the gaseous phase are achieved with a Pfeiffer QME 200 quadrupole mass spectrometer. Gases are transferred to the spectrometer through a capillary tube that is long enough to keep the pressure below $10^{-5} \mathrm{mbar}$ in the spectrometer when the pressure inside the reactor is $0.9 \mathrm{mbar}$. Neutral species are ionized by electron impact at $70 \mathrm{eV}$ in the ionization chamber. The resolution of the spectrometer is 100 at $\mathrm{m} / \mathrm{z} 100$ and it covers the $1-100 \mathrm{u}$ mass range. Most abundant gases and only neutral stable compounds can be detected with this technique. Carbon dioxide consumption is 
Table 1

Composition of the Studied Gaseous Mixture

\begin{tabular}{lccc}
\hline \hline Mixture & {$\left[\mathrm{N}_{2}\right]_{0}(\%)$} & {$\left[\mathrm{CO}_{2}\right]_{0}(\%)$} & {$\left[\mathrm{H}_{2}\right]_{0}(\%)$} \\
\hline 1 & 95 & 1 & 4 \\
2 & 91 & 5 & 4 \\
3 & 86 & 10 & 4 \\
\hline
\end{tabular}

monitored using a time tracking of $\mathrm{CO}_{2}{ }^{+}(\mathrm{m} / \mathrm{z}, 44)$ at a time resolution of $0.5 \mathrm{~s}$.

\subsection{In Situ Infrared Spectroscopy on the Gaseous Phase After In Situ Cold Trapping}

Gaseous species trapped during experiments are analyzed with a Thermo Scientific Nicolet 6700 Fourier Transform Infrared (FTIR) spectrometer. The infrared beam is emitted by the FTIR and passes through the reactor via two $\mathrm{KBr}$ windows. Then the beam is collected by a Mercury Cadmium Telluride detector cooled by liquid nitrogen. In results presented below, IR spectra are recorded in the $650-4500 \mathrm{~cm}^{-1}$ range with a resolution of $1 \mathrm{~cm}^{-1}$ after a co-addition of 500 scans.

\section{RESULTS AND DISCUSSIONS}

\section{1. $\mathrm{CO}_{2}$ Consumption}

The evolution of the $\mathrm{CO}_{2}$ mixing ratio when the plasma is switched on is studied by in situ mass spectrometry. Figure 1 shows this evolution for three different mixing ratios: $1 \%, 5 \%$, and $10 \%$. The initial concentration of $\mathrm{CO}_{2}$ is known, and it is possible to calibrate the $\mathrm{m} / \mathrm{z}, 44$ signal intensity in order to obtain the evolution of the in situ $\mathrm{CO}_{2}$ concentration. The concentration obtained has been multiplied for $1 \%$ and $5 \%$ of $\mathrm{CO}_{2}$ by a factor 10 and a factor 2 , respectively. The $\mathrm{CO}_{2}$ mixing ratio decreases when the plasma is switched on and reaches a steady-state after a transient regime.

We defined the $\mathrm{CO}_{2}$ consumption efficiency $e$ that can be deduced from Figure 1 with Equation (1):

$$
e=\frac{\left[\mathrm{CO}_{2}\right]_{0}-\left[\mathrm{CO}_{2}\right]_{\mathrm{ss}}}{\left[\mathrm{CO}_{2}\right]_{0}} .
$$

Here $\left[\mathrm{CO}_{2}\right]_{0}$ and $\left[\mathrm{CO}_{2}\right]_{\mathrm{ss}}$ represent the initial and steady-state percentages of $\mathrm{CO}_{2}$, respectively. Results are presented in Table 2. The $\mathrm{CO}_{2}$ consumption efficiency has the same order of magnitude for the three initial amounts of $\mathrm{CO}_{2}$ studied. We observed a small decrease of this efficiency for $10 \%$ of $\mathrm{CO}_{2}$ comparatively to $1 \%$ and $5 \%$ with a decrease of $e$ from $24 \%$ to $20 \%$.

\subsection{Water Formation and Quantification}

Panel (a) of Figure 2 presents the evolution of the pressure in the reactor during the increase of the plasma box temperature. We observe an increase of the pressure correlated to the increase of the temperature, which corresponds to the sublimation of the trapped species. The identification of the species released is performed using in situ infrared spectroscopy. Panel (b) of Figure 2 presents two infrared spectra recorded at $173 \mathrm{~K}$ before the release of the species and at $294 \mathrm{~K}$ when the trapped species are released.
We observe an important increase of the water signature in the 1300-2000 and in the $3400-4000 \mathrm{~cm}^{-1}$ ranges.

Water formation can be explained by the production of oxygen radical $\mathrm{O}\left({ }^{1} \mathrm{D}\right)$ by dissociation of $\mathrm{CO}_{2}$ in the plasma:

$$
\mathrm{CO}_{2}+e^{-} \rightarrow \mathrm{CO}+\mathrm{O}\left({ }^{1} \mathrm{D}\right)+e^{-} .
$$

$\mathrm{O}\left({ }^{1} \mathrm{D}\right)$ can be also produced by the dissociative recombination of $\mathrm{CO}_{2}{ }^{+}$(Skrzypkowski et al. 1998; Geppert \& Larsson 2008):

$$
\begin{gathered}
\mathrm{CO}_{2}+e^{-} \rightarrow \mathrm{CO}_{2}^{+}+2 e^{-} \\
\mathrm{CO}_{2}{ }^{+}+e^{-} \rightarrow \mathrm{CO}+\mathrm{O}\left({ }^{1} \mathrm{D}\right) .
\end{gathered}
$$

Then $\mathrm{O}\left({ }^{1} \mathrm{D}\right)$ can react with $\mathrm{H}_{2}$ to from $\mathrm{OH}$ radical:

$$
\mathrm{H}_{2}+\mathrm{O}\left({ }^{1} \mathrm{D}\right) \rightarrow \mathrm{OH}+\mathrm{H} \text {. }
$$

Finally $\mathrm{OH}$ induces the formation of $\mathrm{H}_{2} \mathrm{O}$ by the following reactions:

$$
\begin{gathered}
\mathrm{H}_{2}+\mathrm{OH} \rightarrow \mathrm{H}_{2} \mathrm{O}+\mathrm{H} \\
2 \mathrm{OH} \rightarrow \mathrm{H}_{2} \mathrm{O}+\mathrm{O} .
\end{gathered}
$$

This is in agreement with the modeling of the electron energy distribution functions (EEDF) in our plasma discharge. Indeed, the calculated EEDF realized in pure nitrogen presents a maximum at $2 \mathrm{eV}$ and a relatively populated tail for electron energy above $4 \mathrm{eV}$ (Alves et al. 2012). Thus, the plasma produces electrons with enough energy to produce the above Reactions (R1).

Since $\mathrm{H}_{2} \mathrm{O}$ has absorption bands without overlap of other species, it is possible to estimate its concentration using the Beer-Lambert law where the absorbance $A(\lambda)$ at a given wavelength is defined by

$$
A(\lambda)=\varepsilon(\lambda) \times l \times[C],
$$

where $\epsilon(\lambda)$ is the absorption cross-section of the molecule at a given wavelength, $l$ is the path length of the beam through the gas cell, and $[C]$ is the concentration of absorbing molecules in the reactor:

$$
[C]=\frac{A(\lambda)}{\varepsilon(\lambda) \times l} .
$$

To calculate the concentration of $\mathrm{H}_{2} \mathrm{O}$, we used the absorption cross-section from the HITRAN 2008 database (Rothman et al. 2009). In order to overcome the difference of resolution between the database and laboratory data, we calculate the concentration on the area of the bands. For $\mathrm{H}_{2} \mathrm{O}$, integration is performed both in the $1300-1600$ and in the $3400-4000 \mathrm{~cm}^{-1}$ ranges. Knowing the volume of the reactor, we calculate the number of molecules of water $\mathrm{N}_{\mathrm{H}_{2} \mathrm{O}}$ trapped over four hours. Knowing the gas flow of $\mathrm{CO}_{2}$ injected in the reactor and the consumption efficiency of $\mathrm{CO}_{2}$ for each $\mathrm{CO}_{2}$ initial amounts, we determine the number of $\mathrm{CO}_{2}$ molecules consumed in a four hour duration of the plasma. The consistent values of $\mathrm{N}_{\mathrm{H}_{2} \mathrm{O}}$ obtained for the two ranges are averaged and reported in Table 2 for each $\mathrm{CO}_{2}$ initial amount, such as the number of $\mathrm{CO}_{2}$ molecules consumed, $\mathrm{N}_{\mathrm{CO}_{2}}$.

We observe an important water net production despite a competitive destruction by plasma bombardment resulting from a stationary equilibrium between loss and production pathways in the reactive medium. Table 2 shows an increase, by a factor of two of the number of water molecules produced in the 


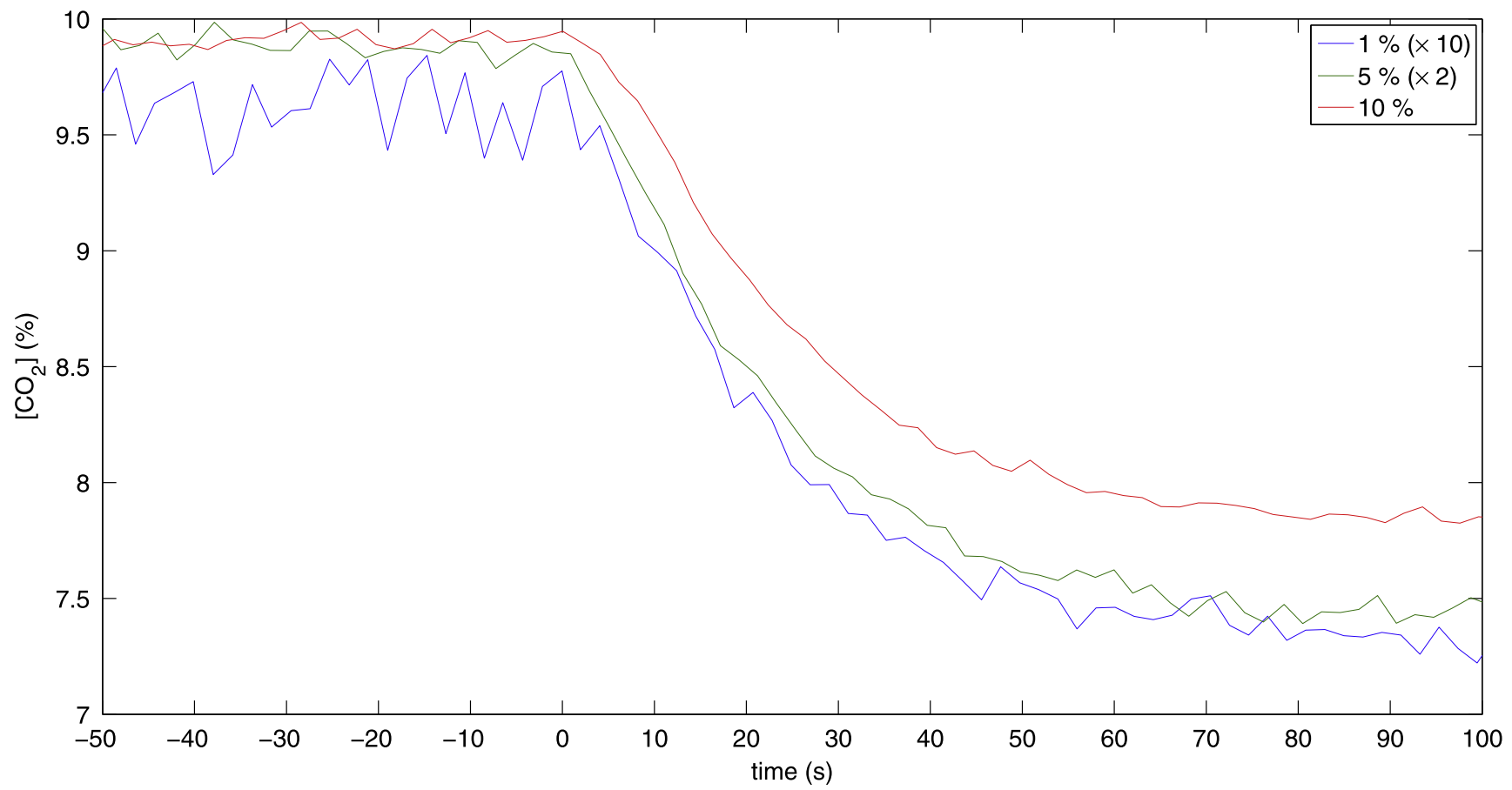

Figure 1. Evolution of $\mathrm{CO}_{2}$ mixing ratio in the gaseous reactive medium with the plasma duration. Origin of time is set as the moment when the plasma is turned on. $\mathrm{CO}_{2}$ mixing ratio has been multiplied by a factor 10 and a factor 2 for an initial amount of $1 \%$ and $5 \%$, respectively.

Table 2

Evolution of the $\mathrm{CO}_{2}$ Consumption Efficiency $e$, the Number of the $\mathrm{CO}_{2}$ Molecules Consumed $\mathrm{N}_{\mathrm{CO}_{2}}$, and the Mean of $\mathrm{H}_{2} \mathrm{O}$ Molecules Formed in Four Hours of Plasma Duration $\mathrm{N}_{\mathrm{H}_{2} \mathrm{O}}$, as a Function of the Initial $\mathrm{CO}_{2}$ Amount

\begin{tabular}{lccc}
\hline \hline$\left[\mathrm{CO}_{2}\right]_{0}(\%)$ & $e(\%)$ & $N_{\mathrm{CO}_{2}}$ (Molecules) & Mean $N_{\mathrm{H}_{2} \mathrm{O}}($ Molecules $)$ \\
\hline 1 & $24 \pm 2$ & $(3 \pm 1) \times 10^{24}$ & $(2.1 \pm 0.1) \times 10^{20}$ \\
5 & $24 \pm 0.6$ & $(4 \pm 1) \times 10^{24}$ & $(3.1 \pm 0.1) \times 10^{20}$ \\
10 & $20 \pm 0.4$ & $(7 \pm 1) \times 10^{24}$ & $(3.9 \pm 0.1) \times 10^{20}$ \\
\hline
\end{tabular}

plasma when the $\mathrm{CO}_{2}$ initial amount increases from $1 \%$ to $10 \%$. This is in good agreement with the increase of the number of $\mathrm{CO}_{2}$ molecules consumed in the plasma, which is multiplied by a factor of three. There is a direct correlation between the quantity of $\mathrm{CO}_{2}$ consumed and the quantity of water produced in agreement with the water formation pathways.

We have first experimentally evidenced that such a water formation could take place in the early Earth's stratosphere from neutral photochemistry and in the ionosphere where $\mathrm{O}$ $\left({ }^{1} \mathrm{D}\right)$ would issue of the dissociative recombination of $\mathrm{CO}_{2}{ }^{+}$. This is compatible with solar flux on the early Earth's atmosphere where a high photon flux in VUV is presumed (Claire et al. 2012). $\mathrm{H}_{2} \mathrm{O}$ is expected to be competitively dissociated by VUV photons, but the efficiency of the production process could ensure a significant stationary water concentration in the atmosphere of the early Earth.

\section{IMPACT FOR THE EARLY EARTH}

\subsection{Water Content in the Early Earth's Atmosphere}

We have experimentally highlighted a source of water located in higher atmospheric layers of the early Earth where $\mathrm{CO}_{2}$ is photodissociated. We provide here a first order estimation of the water concentration in the atmosphere considering this new mechanism. The pressure level of present day Earth's stratopause is chosen as a benchmark for the calculation.

Water concentration is driven by the competition between the production rate $\left(\operatorname{Prod}_{\mathrm{H}_{2} \mathrm{O}}\right)$ and the destruction rate $\left(\operatorname{Loss}_{\mathrm{H}_{2} \mathrm{O}}\right)$,

$$
\operatorname{Prod}_{\mathrm{H}_{2} \mathrm{O}}=\operatorname{Loss}_{\mathrm{H}_{2} \mathrm{O}} \text {. }
$$

Above the stratopause, we roughly assess that the production of $\mathrm{H}_{2} \mathrm{O}$ is limited by the $\mathrm{CO}_{2}$ photodissociation rate, which provides the reactive oxygen atoms. Nowadays, $J_{\mathrm{CO}_{2}}$ is about $10^{-10} \mathrm{~s}^{-1}$. A model study has shown that during the Archean, $J_{\mathrm{CO}_{2}}$ was equal to twice the actual value with a constant value from the stratopause to the atmospheric layers above (Claire et al. 2012). At the stratopause, following the study of Kasting (1993), the partial pressure of $\mathrm{CO}_{2}$ is about $0.1 \mathrm{mbar}$, corresponding to a concentration of $10^{15}$ molecules $\mathrm{cm}^{-3}$. We can deduce the production rate of $\mathrm{H}_{2} \mathrm{O}$ at the stratopause:

$$
\operatorname{Prod}_{\mathrm{H}_{2} \mathrm{O}}=J_{\mathrm{CO}_{2}} \times\left[\mathrm{CO}_{2}\right] \text {. }
$$

The resulting production rate of $\mathrm{H}_{2} \mathrm{O}$ is about $10^{5}$ molecules $\mathrm{cm}^{-3} \mathrm{~s}^{-1}$.

The destruction of $\mathrm{H}_{2} \mathrm{O}$ is equal to the product between the water concentration and the photochemical rate constant, which is the inverse of the lifetime of water $\left(\tau_{\mathrm{H}_{2} \mathrm{O}}\right)$ :

$$
\operatorname{Loss}_{\mathrm{H}_{2} \mathrm{O}}=\left[\mathrm{H}_{2} \mathrm{O}\right] \times\left(1 / \tau_{\mathrm{H}_{2} \mathrm{O}}\right) .
$$

The water concentration is deduced by combining Equations (4)-(6):

$$
\left[\mathrm{H}_{2} \mathrm{O}\right]=\operatorname{Prod}_{\mathrm{H}_{2} \mathrm{O}} \times \tau_{\mathrm{H}_{2} \mathrm{O}}
$$

For a water lifetime of about one day (J. F. Kasting 2015, private communication), a concentration of $10^{10}$ molecules $\mathrm{cm}^{-3}$ is obtained, corresponding to a partial 

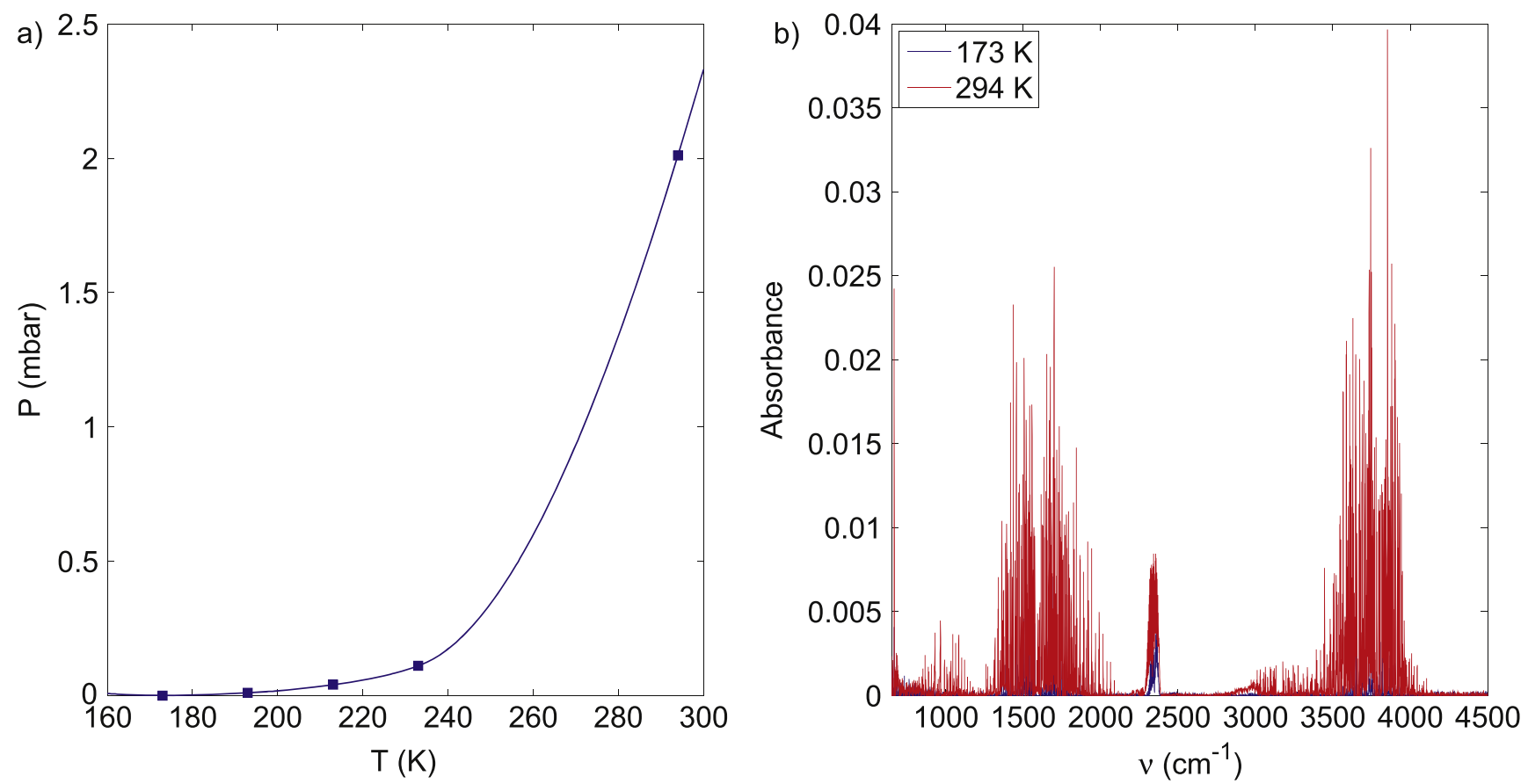

Figure 2. Evolution of the pressure in the reactor during the warm up of the plasma box (on the left) and the evolution of the infrared signature of the gaseous phase in the reactor with an important release of water (on the right).

pressure of water $\sim 10^{-6}$ mbar. This rough calculation informs us on the efficiency of the process with a water value as important at the stratopause $(\sim 50 \mathrm{~km})$ as the ones predicted at the tropopause $(\sim 10 \mathrm{~km})$ in Kasting (1993) without this source.

\subsection{Formation of High Altitude Clouds}

In a $\mathrm{CO}_{2}$-rich atmosphere the average temperature at the stratopause level is expected to be close to the planetary skin temperature, around $200 \mathrm{~K}$ (Kasting 1988), or even below. These low temperature and pressure conditions should result in the solid condensation of any produced water vapor, were the temperature to fall below $\sim 170 \mathrm{~K}$. This would lead to the formation of high altitude water ice clouds, analogous to the present day polar mesospheric clouds (PMC; DeLand et al. 2006; Pérot et al. 2010; Määttänen et al. 2013) whose formation is known to be favored by local production from the methane oxidation (Thomas et al. 1989) or analogous to the present day Polar Stratospheric Clouds (PSC; Lowe \& MacKenzie 2008) if the water production takes place at relatively low altitudes.

These clouds are usually optically thin, but could nevertheless alter Earth's radiation budget in both visible (raising the albedo) and thermal IR radiation (through backscattering of thermal IR radiation), making predictions of their exact influence on the early Earth's climate hard to assess without proper microphysical and radiative modeling, which is beyond the scope of this paper.

These clouds can influence the atmospheric chemistry of the early Earth. Indeed, they are known to play an important role in planetary atmospheres. In the Martian atmosphere, water ice clouds have been found to directly influence the ozone concentration; in the absence of clouds, $\mathrm{OH}$ radicals titrate ozone, whereas heterogeneous chemistry of those radicals on water ice clouds enable ozone transient productions (Lefevre et al. 2008). Similarly in the present Earth's atmosphere, PSC are involved in the stratospheric $\mathrm{O}_{3}$ destruction (Lowe \& MacKenzie 2008).

\subsection{Effect on the Water Atmospheric Profile}

The local extra greenhouse effect of this produced water vapor at stratopause level is almost certainly negligible due to the very low thermal IR opacity of the atmosphere at such low pressures. However, if some water could reach the denser and optically thicker layers at the tropopause level (whether by direct mixing, or sedimentation and sublimation of the aforementioned PMC), possible radiative impacts could be (1) a local cooling of the stratosphere since the extra water vapor is a very effective thermal IR radiator-that is, assuming that a positive temperature anomaly would exist in the first place in an already $\mathrm{CO}_{2}$-rich atmosphere; and (2) a slight increase of the greenhouse $(\sim 0.1 \mathrm{~K}$ assuming a vertically uniform $\mathrm{H}_{2} \mathrm{O}$ mixing ratio above the tropopause), effectively moving the tropopause slightly upwards (on the order of a few tens of meters).

If water production above the stratosphere has a negligible effect on climate, it still impacts the whole atmosphere through chemistry and transport processes. This has been learned with the case of Titan, the largest satellite of Saturn. This object is being observed by the Cassini-Huygens mission that has highlighted the importance of ionospheric chemistry for the production of organic aerosols, impacting the entire atmosphere (Waite et al. 2007). For the early Earth, the presence of water at high altitudes can sensibly modify the concentration of all the other trace species, as $\mathrm{H}_{2} \mathrm{O}$ is known to be a very reactive species at these altitudes. For example, $\mathrm{H}_{2} \mathrm{O}$ can be a source of $\mathrm{NH}$ radicals through its reaction with $\mathrm{N}\left({ }^{2} \mathrm{D}\right)$, produced by photodissociation of $\mathrm{N}_{2}$ at these altitudes (Dobrijevic et al. 2014). Consequences on ammonia $\mathrm{NH}_{3}$ concentration 
in the upper atmosphere of the early Earth can therefore be expected.

\section{CONCLUSION}

This work presents a first experimental study of the reactivity of the early Earth's atmosphere. We used an internal cold trap to analyze the composition of the gaseous phase with a focus on the production of water vapor.

Water is the main product detected in situ by infrared spectroscopy. The evolution of the quantity of water produced as a function of the initial amount of $\mathrm{CO}_{2}$ has been studied. The quantity of water produced increases by a factor of two when the initial amount of $\mathrm{CO}_{2}$ increases from $1 \%$ to $10 \%$.

The important production of water is explained by the production of $\mathrm{O}\left({ }^{1} \mathrm{D}\right)$ radicals by both neutral and ionic pathways. This production highlighted experimentally breaches our knowledge of the Earth's primitive atmosphere where the atmosphere above the troposphere was generally considered as dry, with a sole tropospheric source.

This important production of $\mathrm{H}_{2} \mathrm{O}$ would involve a modification of the water mixing ratio profile with an increase of the water content above the troposphere. Chemistry in these upper atmospheric layers has therefore to be revisited. The increase of the water content in the stratosphere and mesosphere could notably result in the formation of high altitude clouds similar to actual PSC and PMC, whose major impact would be the development of heterogeneous processes and chemistry. Further modeling studies are required to conclude on the overall chemical and climate resulting impact of such a water source above the troposphere.
B. F. acknowledges the Île-de-France region (DIM ACAV) for his thesis funding. The authors also wish to thank J. F. Kasting for his precise comments during the review process that led to significant improvements to this paper.

\section{REFERENCES}

Alcouffe, G., Cavarroc, M., Cernogora, G., et al. 2010, PSST, 19, 015008 Alves, L. L., Marques, L., Pintassilgo, C. D., et al. 2012, PSST, 21, 045008 Claire, M. W., Sheets, J., Cohen, M., et al. 2012, ApJ, 757, 95

DeLand, M. T., Shettle, E. P., Thomas, G. E., \& Olivero, J. J. 2006, JASTP, 68,9

Dobrijevic, M., Hébrard, E., Loison, J. C., \& Hickson, K. M. 2014, Icar, 228,324

Geppert, W. D., \& Larsson, M. 2008, MolPh, 106, 2199

Kasting, J. 1993, Sci, 259, 920

Kasting, J. F. 1988, Icar, 74, 472

Kuramoto, K., Umemoto, T., \& Ishiwatari, M. 2013, E\&PSL, 375, 312

Lefevre, F., Bertaux, J.-L., Clancy, R. T., et al. 2008, Natur, 454, 971

Lowe, D., \& MacKenzie, A. R. 2008, JASTP, 70, 13

Määttänen, A., Pérot, K., Montmessin, F., \& Hauchecorne, A. 2013, in Comparative Climatology of Terrestrial Planets, ed. S. J. Mackwell et al. (Tucson, AZ: Univ. Arizona Press), 393

Marty, B., Zimmermann, L., Pujol, M., Burgess, R., \& Philippot, P. 2013, Sci, 342, 101

Pérot, K., Hauchcorne, A., Montmessin, F., et al. 2010, ACP, 10, 2723

Rothman, L. S., Gordon, I. E., Barbe, A., et al. 2009, JQSRT, 110, 533

Skrzypkowski, M. P., Gougousi, T., Johnsen, R., \& Golde, M. F. 1998, JChPh, 108,8400

Szopa, C., Cernogora, G., Boufendi, L., Correia, J. J., \& Coll, P. 2006, P\&SS, 54,394

Thomas, G. E., Olivero, J. J., Jensen, E. J., Schroeder, W., \& Toon, O. B. 1989, Natur, 338, 490

Waite, J. H., Young, D. T., Cravens, T. E., et al. 2007, Sci, 316, 870

Wordsworth, R. D., \& Pierrehumbert, R. T. 2013, ApJ, 778, 154 